Volume 9, No.1, January - February 2020

International Journal of Advanced Trends in Computer Science and Engineering

Available Online at http://www.warse.org/IJATCSE/static/pdf/file/ijatcse78912020.pdf

https://doi.org/10.30534/ijatcse/2020/78912020

\title{
An Improved Weighted KNN Algorithm for Indoor Positioning using Beacon Devices
}

\author{
Chuan-Bi Lin ${ }^{1}$, Jong-Shin Chen ${ }^{2}$, Yong-Yu Peng ${ }^{3}$, Yung-Fa Huang ${ }^{4 *}$ \\ ${ }^{1}$ Chaoyang University of Technology, Taiwan, cblin@ cyut.edu.tw \\ ${ }^{2}$ Chaoyang University of Technology, Taiwan, jschen26@ cyut.edu.tw \\ ${ }^{3}$ Chaoyang University of Technology, Taiwan, s10630612@gm.cyut.edu.tw \\ ${ }^{4}$ Chaoyang University of Technology, Taiwan, yfahuang@ cyut.edu.tw \\ *Corresponding author: yfahuang@cyut.edu.tw
}

\begin{abstract}
To avoid the problem of interference and improve the accuracy of indoor positioning is a hot topic. In this paper, we proposed a weighted K-nearest neighbor (WKNN) algorithm for indoor positioning with The received signal strength indication (RSSI) from Beacon Devices, in which 9 fixed locations are arranged for indoor positioning experiments. The received signals are smoothed by the Kalman filter and moving average windows. Then the positioning procedures were performed by the KNN algorithm and the proposed WKNN. Experimental results show that the proposed WKNN can outperform the KNN method with $83 \%$ accuracy.
\end{abstract}

Key words : Location Based Services, Indoor Positioning, Beacon, Fingerprinting, Received Signal Strength Indication.

\section{INTRODUCTION}

With the rapid development of modern technology in information and communication technology, Location Based Services (LBSs) have been widely used to make lives more intelligent and convenient [1]. The indoor positioning technology plays an important role among LBSs to provide more service applications. Global Positioning System (GPS) [2,3] is one of positioning technologies and is widely used in various outdoor scenarios such as transportation navigation and logistics systems. However, the GPS signal is easily affected by the building and the satellite signal is greatly affected, and they cannot be used indoors. Thus we need to use indoor positioning technology.

Indoor positioning uses short-range wireless communication technologies, such as: WiFi (Wireless Fidelity) [4], Bluetooth Low Energy (BLE) [5] and other wireless communication technologies. Among them, WiFi positioning and BLE positioning are used by many people. Although $\mathrm{WiFi}$ positioning has fast transmission speed and long transmission distance, the power consumption and cost of WiFi positioning are higher than BLE positioning. BLE has low cost and low power consumption characteristics. Moreover, BLE is a standard proposed by the Bluetooth Special Interest Group and is applicable to Bluetooth version 4.0.
Beacon is a device that uses Bluetooth technology to transmit signals to achieve indoor positioning. It has the characteristics of low cost and small size, and can broadcast various messages as long as it is within the signal range. The triangular positioning method used in indoor positioning is to convert the signal strength into a distance for positioning. However, it easily causes the positioning accuracy to decrease due to the movement of people in the environment or the signal attenuation caused by the equipment. The fingerprint positioning method [6] collects different the received signal strength indication (RSSI) of the location, which is treated as the signal characteristic of each location and stored in the fingerprint database. Then by comparing the received new signal strength with the fingerprint database, the current location can be decided [7].

The fingerprint positioning method in indoor positioning can be divided into two phases: the training phase and the positioning phase. In the training phase, we need to collect the fingerprints of the positioning area to establish a database. During the positioning phase, we measure the RSSI value and compare the maps. Therefore, in order to reduce the instability of the signal, the signal of the fingerprint localization method [8] is positioned by K-nearest neighbor (KNN) algorithm.

The KNN algorithm is based on actual examples to make decisions. Select the nearest node to compare the number and judge. However, the above method may lead to a low positioning error tolerance, because the signal may have outliers and the algorithm does not consider the weight problem. Therefore, in this paper, after the signal passes through $\mathrm{KF}$, the machine learning WKNN algorithm is used to increase the accuracy of BLE indoor positioning. The experimental method is with indoor space. Therefore, this research will conduct indoor positioning experiments, complete Beacon sensor and receive signal equipment to complete the indoor positioning system.

\section{RELATED WORKS}

\subsection{Wireless Communications}

In recent years, wireless sensor networks (WSNs) have been continuously developed due to advances in wireless 
communication technology, microcontrollers, and battery technology. WSN places various sensors in the environment. These nodes then use wireless communication technology to pass the collected data to the server for calculation. WSNs can be applied in the fields of smart home, health detection, and environmental monitoring [9]. The sensing network formed by the Beacon equipment in this paper through Bluetooth transmission technology is one of the applications of WSN.

The WiFi is a wireless local area network technology established by the IEEE 802.11 standard. Wireless routers, wireless network cards and other devices are formed by devices that meet the standard certification. WiFi positioning [10] is suitable for indoor positioning in a small area.

The Beacon is a transmitter of broadcast signals. It uses low-power Bluetooth technology to broadcast the signal strength and continuously emits its own signals. The signals include unique identification codes, major numbers, and minor numbers as identification information [11]. Any Micro-location signal transmitters using low-power Bluetooth technology can be called Beacon. When the user turns on the Bluetooth of the smart phone and enters the Beacon broadcast signal range, the received signal strength value will change with the distance, and the relevant information is used to determine the user's current location.

The Bluetooth technology was proposed by Ericsson in 1994, established the Bluetooth Special Interest Group (SIG), and formulated the Bluetooth technology standard. Bluetooth is a wireless communication protocol that belongs to short-distance transmission technology. The Bluetooth 4.0 version was released in June 2010. The Bluetooth 4.0 technology is compatible with traditional Bluetooth and high-speed Bluetooth. In addition, a new technology is added for Bluetooth Low Energy (BLE). The BLE [12] is suitable for applications with less bandwidth.

\subsection{Positioning Methods}

Wireless communication technology is used as the basis for indoor positioning. The transmitter is set up in the indoor space. The signal of the transmitter or the distance is converted into the distance to calculate the indoor space position. The following is three methods. Triangular positioning mainly uses the signal strength of the transmitter to calculate the distance from the receiving end. The triangular positioning method must allow the device at the receiving end to receive the signal strength of more than three transmitters and to obtain the user's current position by the minimum mean square error (MMSE) method [10].

The fingerprint positioning method has different values of the received signal strength at different locations in the room, thereby establishing fingerprints at each location in the room. The signal strength collected in real time is compared with the fingerprint database according to the algorithm to achieve positioning. The fingerprint positioning method can be divided into two phases [13], the training phase and the positioning phase. The main purpose of the training phase is to establish a location fingerprint database before the positioning phase. According to the distribution of indoor environment design nodes, the signal strength of each node is collected, and the location information is recorded in the database later. During the positioning phase, the smart phone or smart device is used to scan the Beacon signal in the environment to receive the signal strength (Received Signal Strength Indication, After the RSSI value is uploaded to the server, it is compared with the fingerprint database stored in the training stage, and the current location information is returned to the user after calculation by the algorithm.

\subsection{KNN Algorithms}

The KNN [14] algorithm is an instance-based algorithm. The concept is an example of the same category and the similarity between them is high. Similarity, it can evaluate the possible classification of cases of unknown category. The KNN will choose the nearest $K$ samples in space to predict the results based on the nature of the samples, as shown in Fig. 1, where $\mathrm{K}$ indicates that the $\mathrm{K}$ nearest distance classification is selected for decision making, and the circle indicates classification A and the star indicates classification B. When $K=3$, the new data will select the 3 closest data. Because most of the data are classification B, the new data is classified as a classification B.

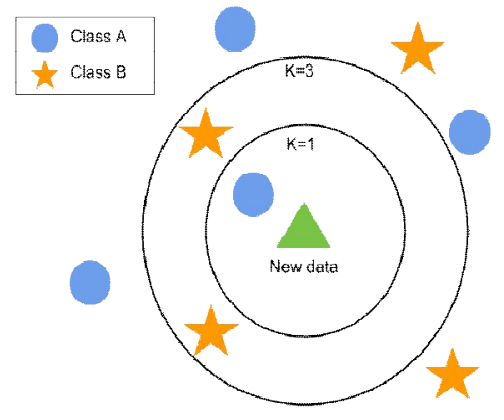

Figure 1: KNN methods

\section{POSITIONING SYSTEMS}

The positioning architecture is shown in Fig. 2. In this study, the fingerprint positioning method is used for positioning. The Beacon device transmits signals through the Bluetooth transmission protocol. Users' equipments received the signal 's RSSIs. Before positioning procedure, the RSSI signals will be collected. The RSSIs of each location in the room can be as a fingerprint. The RSSI value has different values at different locations. Therefore, it can be used as a basis for determining the location. The RSSI value is stored in the server, and the server side receives the unclassified RSSI value. It is brought 
into the WKNN algorithm for calculation and is compared with the previous data stored in the server for fingerprint comparison. Fingerprints established at different locations can calculate the user's classified location

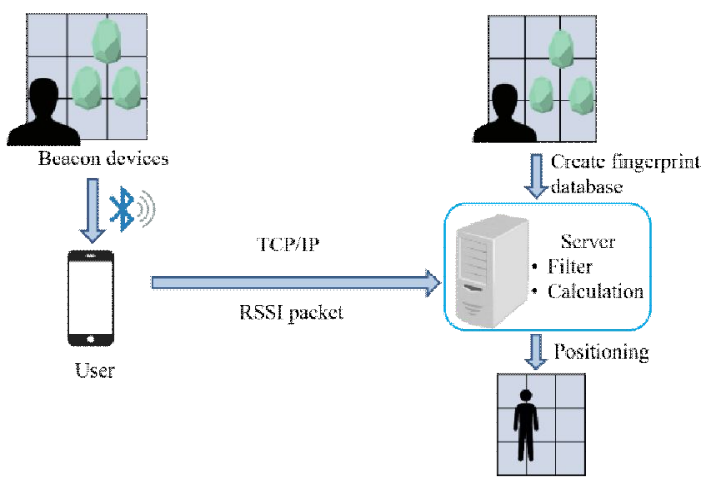

Figure 2: Positioning methods procedures

The Beacon device is like a small microcomputer. With low-power Bluetooth technology, it continuously broadcasts signal to be received by Bluetooth-capable devices, such as smartphones. After the wireless signal is transmitted from the transmitting device, the receiving end receives the signal. Then it propagates in all directions in the environment. In the wireless communication channels, there are many factors that will affect the transmission of wireless signals such as distance and environmental settings. The received signal power, which is a ratio based on $1 \mathrm{~mW}$ power, can be expressed by

$$
\operatorname{RSSI}(\mathrm{dBm})=10 \times \log _{10}\left(\frac{P_{r}}{1 \mathrm{~mW}}\right)
$$

Where $\mathrm{Pr}$ is the received power with the unit $\mathrm{mW}$.

Kalman filter is an optimized autoregressive data processing algorithm [15], which has been widely used in various industries. Because the original signal value will be unstable due to various interferences, the signal value needs to be smoothed by the Kalman filter. The operation mode of the Kalman filter has two phases: the prediction phase and the update phase. At prediction phase, the estimated value of the current state is predicted according to the estimated value $x_{t-1}$ of the previous state, which can be expressed as

$$
x_{t}=x_{t-1}
$$

where $x_{t}$ is the estimated value at time $t$, and the prediction error at time $t$ is expressed by

$$
P_{m_{t}}=P_{m_{t-1}}+Q
$$

At updating phase, the observed value of the current state is used to update the estimated value in the prediction phase. The updating procedures can be expressed by

$$
\begin{aligned}
& K_{t}=P_{m_{t}} \div\left(P_{m_{t}}+R\right), \\
& x_{t}=x_{t}+K_{t} \times\left(z_{\mathrm{t}}-x_{t}\right)
\end{aligned}
$$

and

$$
P_{m_{t}}=\left(1-K_{t}\right) \times P_{m_{t}}
$$

where $K_{t}$ is the gain at time $t, z_{t}$ is the measured value at time $t$, and the variables $Q$ and $R$ determine whether the value deviates from the estimated value or the measured value.

In order to reduce multipath fading effect, a moving average window is adopted to remove the variation [16]. The sampling data after the proposed moving window can be expressed by

$$
x_{t}=\frac{1}{L} \sum_{i=t}^{t+L} x_{i}
$$

where $L$ is the window length. We perform the experiments to improve the performance by signal smoothing with moving windows $L=5$.

The WKNN [17] algorithm is based on an extension of the KNN algorithm. KNN uses the majority of the $\mathrm{K}$ nearest samples to determine the final result. The WKNN algorithm will give the weights for the selected samples according to the inverse of the Euclidean distance [18].

In the experimental environment, each mobile device can receive signals from 3 Beacons, so each record is composed of 3 signals. In this method, we first collect enough records for 9 different locations. Let a record $\mathbf{r}_{\mathbf{k}}$ be as

$\mathbf{r}_{k}=\left(x_{k, 1}, x_{k, 2}, x_{k, 3}\right)$

where $x_{i, 1}, x_{i, 2}$, and $x_{i, 3}$ are the 3 signals received from 3 Beacons. Moreover, Let $L\left(\mathbf{r}_{i}\right)$ be the corresponding location of $\mathbf{r}_{i}$. All records are divided into sets $C$ and $T$, where $C$ is the classified data and $T$ is the test data. For any two records $r_{i}$ and $\mathbf{r}_{i}$, their distance is as shown in

$$
D\left(\mathbf{r}_{\mathbf{i}}, \mathbf{r}_{\mathbf{j}}\right)=\sqrt{\left(x_{i, 1}-x_{j, 1}\right)^{2}+\left(x_{i, 2}-x_{j, 2}\right)^{2}+\left(x_{i, 3}-x_{j, 3}\right)^{2}}
$$

The KNN can be described as follows. For any record $\boldsymbol{r}_{i}$ in $T$, three steps are performed to predict its position.

Step 1: For each record $\mathbf{r}_{j}$ in $C$, the distance $D\left(\mathbf{r}_{i}, \mathbf{r}_{j}\right)$ is evaluated.

Step 2: Find the $\mathrm{K}$ records, $\mathbf{r}_{1}^{\prime}, \mathbf{r}_{2}^{\prime}, \ldots, \mathbf{r}_{\mathrm{K}}^{\prime}$, where the distances to $\mathbf{r}_{i}$ are smallest.

Step 3: The classification values $P\left(\mathbf{r}_{1}^{\prime}\right), P\left(\mathbf{r}_{2}^{\prime}\right), \ldots, P\left(\mathbf{r}_{\mathrm{K}}^{\prime}\right)$ of the $\mathrm{K}$ records in Step 2 are used for statistics. The predicted classification of $\mathbf{r}_{i}$ are voted by $P\left(\mathbf{r}_{1}^{\prime}\right), P\left(\mathbf{r}_{2}^{\prime}\right), \ldots, P\left(\mathbf{r}_{\mathrm{K}}^{\prime}\right)$.

In WKNN, $\mathbf{r}_{1}^{\prime}, \mathbf{r}_{2}^{\prime}, \ldots, \mathbf{r}_{\mathrm{K}}^{\prime}$ are first grouped according to their positions $P\left(\mathbf{r}_{1}^{\prime}\right), P\left(\mathbf{r}_{2}^{\prime}\right), \ldots, P\left(\mathbf{r}_{\mathrm{K}}^{\prime}\right)$. Records in the same group will be evaluated their value by summating the inverse distances. Record $\mathbf{r}_{i}$, then are classified into the group with the largest summated value. 


\section{EXPERIMENTAL RESULTS}

\subsection{Experimental Environments}

The experimental environment was conducted at a laboratory. At the laboratory, the length is 6.2 meters, the width is 6.5 meters, and the height is 3 meters. The Beacon equipments are used to broadcast signals and allow mobile phones to receive signal strength. The indoor positioning experiments are performed at a laboratory shown in Fig. 3. In experimental area, RSSI signals are collected at 9 different locations in the area, which is shown as $T_{1}$ to $T_{9}$. From Fig. 4 , it can be seen that between two nearby test positions, it is 1.5 meters apart, and B1, B2, and B3 indicate the placement of the Beacon equipment.

The positioning fingerprint positioning method is used in the positioning process. It needs to go through the training phase and the positioning phase. During the training phase, the indoor space is divided into different classification areas. Due to the signal strength characteristics, there are different signal strengths in different areas. Three groups of RSSI signals are sent. The intensity is used as the fingerprint of the position. After collecting the signal intensity in each classification area, it is transmitted to the back-end server for storage and becomes a fingerprint database. The positioning phase is shown in Fig. 5.

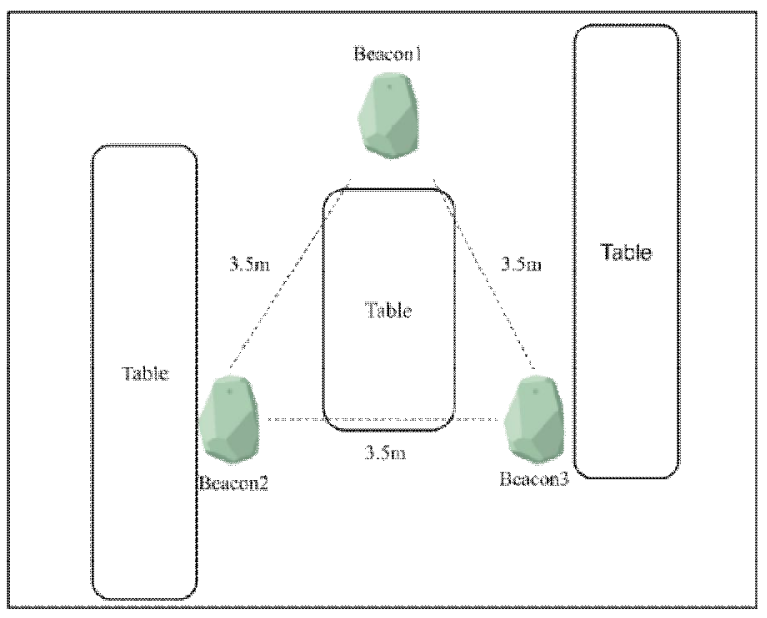

Figure 3: The deployment of three Beacons

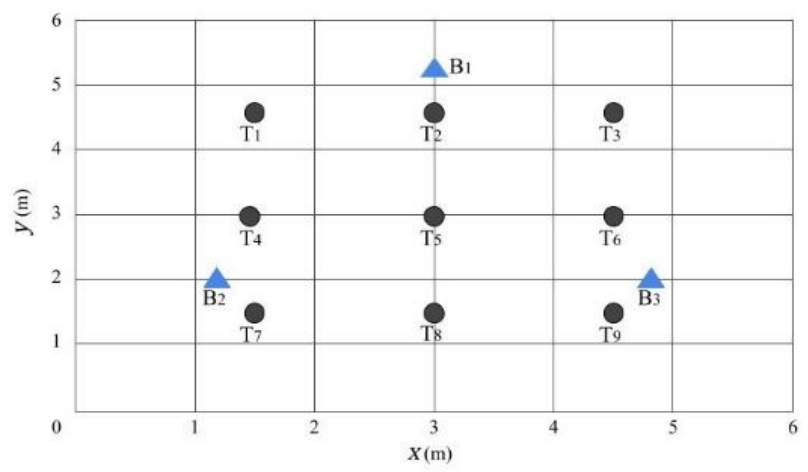

Figure 4: The area of nine testing positions in a laboratory

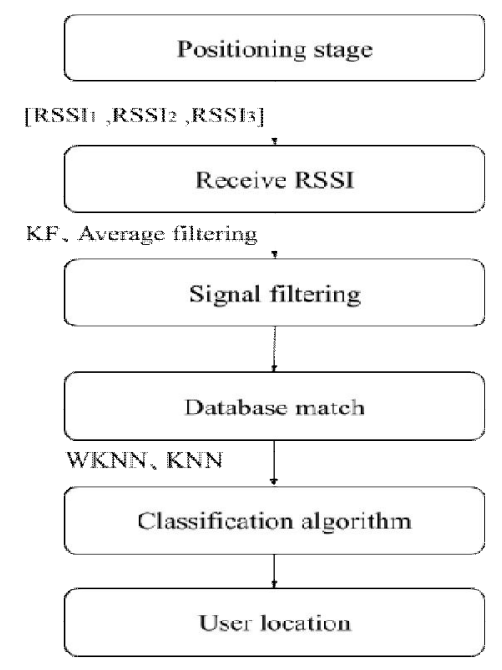

Figure 5: The procedure of positioning phases

In the space, a mobile phone is used to measure three sets of RSSI signals, and the RSSI values are subjected to Kalman filtering or average filtering to reduce signal instability. After filtering, the fingerprint database and the previously established fingerprint database are used. The matching operation is performed. When using the WKNN algorithm for matching, the weights are assigned based on the Euclidean distance ordering and then compared to obtain the positioning result.

Due to the multipath fading effect in wireless communication channel especially for indoors, at first we investigated the RSSI variation of the received signal. Then the Kalman filtering and moving average filtering are applied to smooth the RSSI signals.

The RSSI value of received signal Using Beacon equipment at a distance of 1 meter are sampled 30 samples with sampling rate 0.5 samples per second as shown by original signal in Fig. 6. After the Kalman filter is applied as (2)-(6), the RSSI signal is shown by KF-based in Fig. 6. The estimation is based on the gain, $K$ in (3) and (4). The QR ratio is set to 1:5. Then from Fig. 6, it is observed that the signal after Kalman filtering, the signal is become more stable comparing the original signal.

Moreover, to further stabilize the RSSI of the signal, the moving average filter in (7) is applied after the Kalman filtering. Then from Fig. 6, it is observed that the filtering results with the hybrid-based are definitely more smoothing than both of the original and the KF-based. 


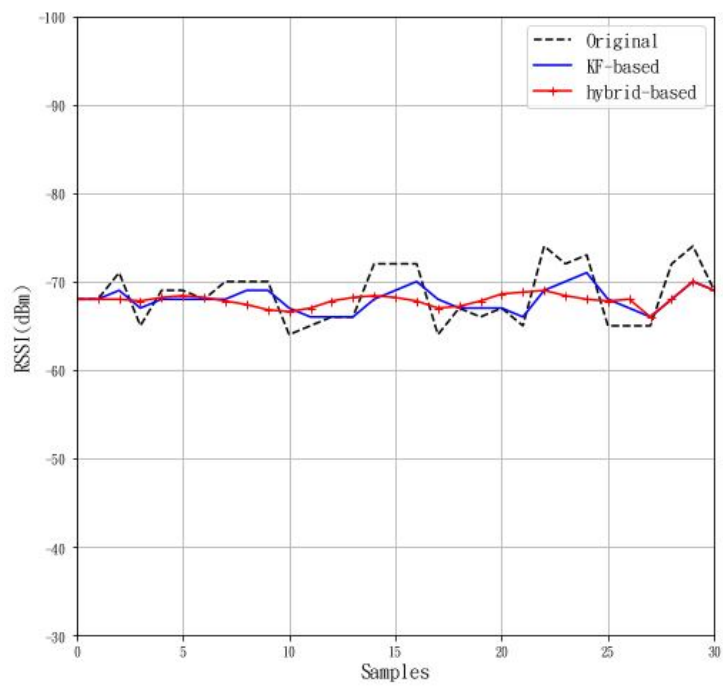

Figure 6: Comparisons of the original received RSSI and the filtering RSSI of the sampled signal

\subsection{KNN Positioning}

In this Section, RSSI signals are used for indoor positioning performed in the laboratory. The Beacon device is used to send RSSI signals, and the RSSI signals are filtered. The accuracy of the positioning in the laboratory is compared with the KNN algorithm.

Total 300 test samples were collected at three different date each time. The 300 records were taken during the training phase. The test site was divided into 9 test positions, and each point was 1.5 meters apart as shown in Fig. 3 . The 300 RSSI signals were collected at each position and tested 3 times at different time, as shown in Figs. 7 and 8, where the average accuracy comes from the average of the accuracy of the 9 test positions ( $T_{1}$ to $\left.T_{9}\right)$.

In the case of using the KNN algorithm for positioning, the accuracy of the RSSI signal in KF filtering and hybrid filtering (Kalman filtering and moving average filtering) is shown in Fig. 7. It can be seen from the Fig. 7 that with the hybrid filtering, a better accuracy between $75 \%$ and $80 \%$ can be obtained.

\subsection{WKNN Positioning}

Moreover, the proposed WKNN algorithm is further used for positioning. The accuracy of the RSSI signal in KF filtering and hybrid filtering is shown in Fig. 8. In addition, 300 RSSI signals were collected at each location, and the KF filtering and KF mixed average filtering were tested 3 times at a QR ratio of 1:5. It can be seen from Fig. 8 that in the case of KF filtering plus moving average filtering (Hybrid), and a better accuracy between $80 \%$ and $85 \%$ can be obtained. The average accuracy comes from the average of the accuracy of the 9 test positions $\left(T_{1}\right.$ to $\left.T_{9}\right)$.

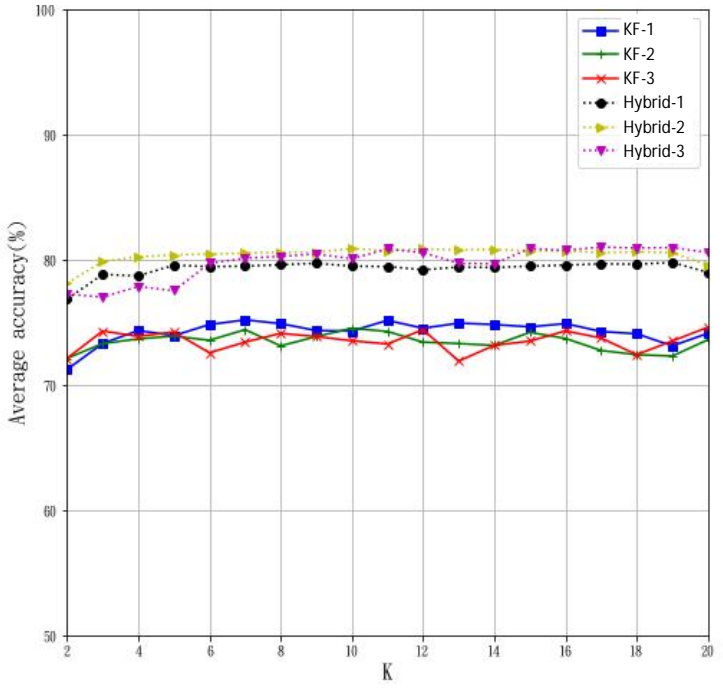

Figure 7: Accuracy of positioning of KNN methods with KF and hybrid filtering

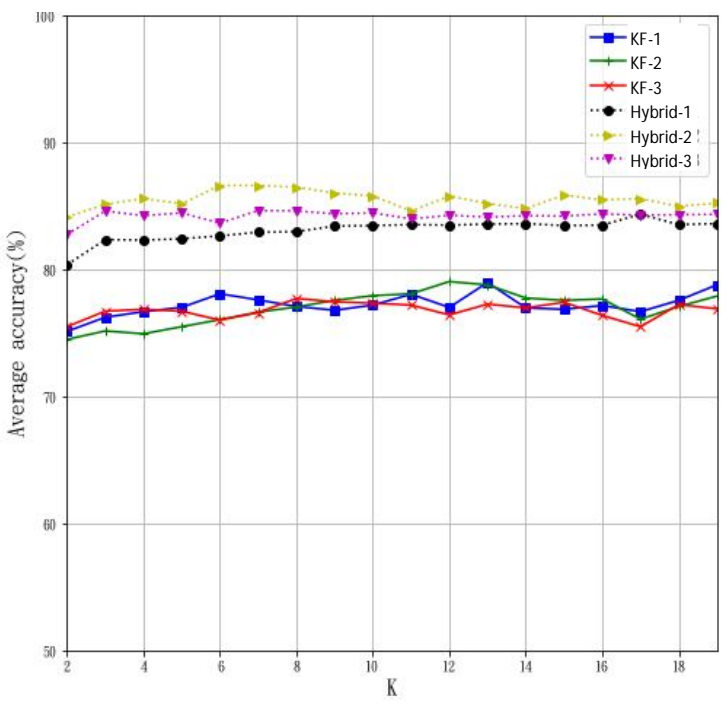

Figure 8: Accuracy of positioning of WKNN methods with KF and hybrid filtering

\subsection{Comparisons of Positioning Accuracy}

In this study, the proposed hybrid filtering of Kalman filtering and moving average windows can effectively reduce the signal variations. Some experiments had been performed. The comparisons of the positioning accuracy of the KNN and WKNN methods combine with the RSSI of original signal, KF filtering and the hybrid filtering are shown in Table 1. From Table 1, it can be seen that when the proposed WKNN uses hybrid filtering, a highest positioning accuracy with $83.5 \%$ can be reached. 
Table 1: Comparisons of Positioning Accuracy for KNN and WKNN

\begin{tabular}{|c|c|c|}
\hline $\begin{array}{c}\text { Signal } \\
\text { Filtering }\end{array}$ & $\begin{array}{c}\text { Positioning } \\
\text { Methods }\end{array}$ & Accuracy \\
\hline \multirow{2}{*}{ Original } & KNN & $63.8 \%$ \\
\cline { 2 - 3 } & WKNN & $66.7 \%$ \\
\hline Kalman & KNN & $73.1 \%$ \\
\cline { 2 - 3 } Filtering & WKNN & $76.4 \%$ \\
\hline Hybrid & KNN & $79.1 \%$ \\
\cline { 2 - 3 } Filtering & WKNN & $83.5 \%$ \\
\hline
\end{tabular}

\section{CONCLUSION}

This paper proposed an indoor positioning system based on the Beacon device. In the case of the original signal, a KNN algorithm is used, and the positioning accuracy is $63.8 \%$. To reduce the signal variation of wireless communications, the received signals are filtered by Kalman and moving average windows. The experimental results show that with the proposed hybrid filtering, the accuracy of indoor positioning with the proposed WKNN is improved to $83.5 \%$.

\section{ACKNOWLEDGEMENT}

The results of this research are sponsored by the Ministry of Science and Technology (MOST), Taiwan, with the grant number is MOST 108-2221- E-324- 010- and 108-2637-E-324-004.

\section{REFERENCES}

1. G. Gartner and H. Huang. Progress in Location-Based Services 2014, 1st ed. Springer International Publishing, 2015. https://doi.org/10.1007/978-3-319-11879-6

2. S. Sananmongkhonchai, P. Tangamchit and P. Pongpaibool. Cell-based traffic estimation from multiple GPS-equipped cars, in Proc. IEEE TENCON, Singapore, 2009.

https://doi.org/10.1109/TENCON.2009.5395897

3. A. Chandra, S. Jain and M. A. Qadeer. GPS locator: An application for location tracking and sharing using GPS for Java enabled handhelds, in Proc. International Conference on Computational Intelligence and Communication Networks, Gwalior, India, 2011. https://doi.org/10.1109/CICN.2011.85

4. M. Ohta, J. Sasaki, S. Takahashi and K. Yamashita. WiFi positioning system without AP locations for indoor evacuation guidance, in Proc. IEEE 4th Global Conference on Consumer Electronics (GCCE), Osaka, Japan, 2015. https://doi.org/10.1109/GCCE.2015.7398642

5. S. Memon, M. M. Memon, F. K. Shaikh and S. Laghari. Smart indoor positioning using BLE technology, in Proc. 4th IEEE International Conference on Engineering
Technologies and Applied Sciences (ICETAS), Salmabad, Bahrain, 2017.

6. L. Qianqian, X. Yubin, Z. Mu, D. Zhi-an and L. Yao. Characteristics of fingerprint location technology in WLAN environment, in Proc. International Forum on Information Technology and Applications, Chengdu, China, 2009.

https://doi.org/10.1109/IFITA.2009.492

7. Z.-G. Du, J.-S. Pan, S.-C. Chu, H.-J. Luo, and P. Hu. Quasi-affine transformation evolutionary algorithm with communication schemes for application of RSSI in wireless sensor networks, IEEE Access, Vol. 8, pp. $8583-8594,2020$.

8. S. N. Duong, A. V. Trinh and T. Dinh. Bluetooth low energy based indoor positioning on iOS platform, in Proc. IEEE 12th International Symposium on Embedded Multicore/Many-core Systems-on-Chip (MCSoC), Hanoi, Vietnam, 2018.

9. Y. Zhang, L. Sun, H. Song and X. Cao. Ubiquitous WSN for healthcare: recent advances and future prospects, IEEE Internet of Things Journal, Vol. 1, No. 4, pp. 311-318, Aug. 2014. https://doi.org/10.1109/JIOT.2014.2329462

10. Y.-F. Huang, Y.-T. Jheng, J.-S. Chen, C.-C. Wei, Y.-J. Cheng and C.-B. Lin. Indoor positioning with reference nodes selection in wireless networks, in Proc. of 2018 International Symposium on Computer, Consumer and Control (IS3C 2018), Taichung, Taiwan, 2018, pp. 445- 448.

11. C.-B. Lin, Y.-F. Huang, L.-X. Chen, and J.-M. Hong. Implementation of a fuzzy localization system based on radio-frequency identification technology for elderly people, Sensors and Materials, Vol. 31, No. 2, pp. 559-567, Feb. 2019.

https://doi.org/10.18494/SAM.2019.2132

12. G. Carles, J. Oller, and J. Paradells. Overview and evaluation of Bluetooth low energy: an emerging low-power wireless technology, Sensors, Basel, Switzerland, Vol. 12, No. 9, pp. 11734-11753, Aug. 2012. https://doi.org/10.3390/s120911734

13. T. De Schepper, A. Vanhulle and S. Latré. Dynamic BLE-based fingerprinting for location-aware smart homes, in Proc. IEEE Symposium on Communications and Vehicular Technology (SCVT), Leuven, Belgium, 2017. https://doi.org/10.1109/SCVT.2017.8240316

14. C. Dong, P. P. K. Chan, W. W. Y. Ng and D. S. Yeung. 2-Stage instance selection algorithm for KNN based on nearest unlike neighbors, in Proc. International Conference on Machine Learning and Cybernetics, Qingdao, China, 2010.

https://doi.org/10.1109/ICMLC.2010.5581078

15. Q. Li, R. Li, K. Ji and W. Dai. Kalman filter and its application, in Proc. 8th International Conference on Intelligent Networks and Intelligent Systems (ICINIS), Tianjin, China, 2015.

16. Y.-F. Huang, Y.-H. Hsu, C.-C. Chang, S.-H. Liu, C.-C. Wei, T.-Y. Yao, and C.-B. Lin. An improved sleep posture recognition based on force sensing resistors, 
In book: Intelligent Information and Database Systems, ACIIDS 2017, Part II, Lecture Notes in Computer Science, Vol. 10192, Springer-Verlag Berlin Heidelberg, Kanazawa, Japan, 2017, pp. 318-327. https://doi.org/10.1007/978-3-319-54430-4_31

17. K. Chernoff and M. Nielsen. Weighting of the k-nearest-neighbors, in Proc. 20th International Conference on Pattern Recognition, Istanbul, Turkey, 2010.

https://doi.org/10.1109/ICPR.2010.168

18. D. Zou, W. Meng and S. Han. Euclidean distance based handoff algorithm for fingerprint positioning of WLAN system, in Proc. IEEE Wireless Communications and Networking Conference (WCNC), Shanghai, China, 2013. 